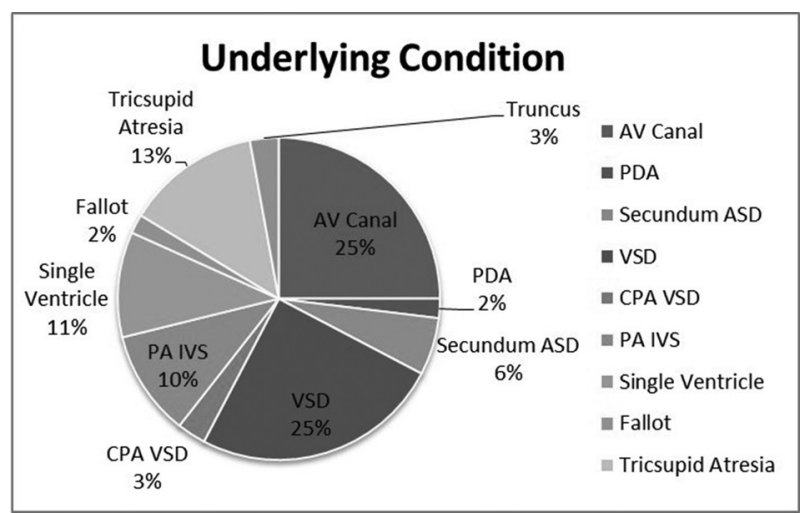

Abstract 74 Figure 1

\section{INHERITED LEFT VENTRICULAR NON-COMPACTION IN SPONTANEOUS MONOCHORIONIC DIAMNIOTIC TWIN PREGNANCY}

${ }^{1} J$ oann Hale, ${ }^{1}$ Rajeswari Parasuraman, ${ }^{2}$ Andrew Curry, ${ }^{1}$ David Howe, ${ }^{2}$ Samantha Fitzsimmons*. ' Wessex Fetal Medicine Unit; ${ }^{2}$ University Hospital Southampton

\subsection{6/heartjnl-2017-311726.74}

Introduction Pregnancy in Left Ventricular Non-Compaction (LVNC) can increase the risk of heart failure, arrhythmias and systemic embolic events. LVNC carries an autosomal dominant trait and incomplete penetrance. This case study demonstrates the importance of including genetic inheritance within prepregnancy counselling, multidisciplinary care and early detection of clinical deterioration in women with LVNC. Whilst we believe this is the first case study with identified genetic testing with an alteration in gene MYH7.

Case report A 27 year old female with a pre-existing diagnosis of LVNC was referred to the high risk pregnancy service at the University Hospital Southampton, from out of region, at 23 weeks gestation with spontaneous monochorionic diamniotic twins. Initial transthoracic echo (TTE) at 23 weeks gestation demonstrated a dilated and heavily trabeculated left ventricle (LV) at $5.6 \mathrm{~cm}$ with severe $\mathrm{LV}$ systolic dysfunction, ejection fraction (EF) 20\%-25\%. The referral pre-pregnancy EF was quoted at 30\%-35\%. Medical therapy was up-titrated (beta-blockade and diuretics) and patient remained NHYA II.

At 27 weeks gestation the patient presented with a nocturnal cough. Clinical examination demonstrated an elevated jugular venous pressure, worsening mitral and tricuspid regurgitation, peripheral oedema and $\mathrm{S}_{3}$. Repeat TTE demonstrated a further deterioration in LV function EF 10\%-15\%, with worsening tricuspid regurgitation and elevated pulmonary artery pressure. The patient was admitted for intravenous diuretics and medication optimisation.

Fetal echocardiogram confirmed the monochorionic twins had inherited the left ventricular non-compaction.

The patient was delivered at 30 weeks by caesarean section in cardiac theatres under general anaesthetic with vascular access in place to allow prompt E-CRP if required.

Postnatally, the patient returned to NYHA II with a 8 month post partum left ventricular EF 30\%-35\%.

Day 1 TTE of the monochorionic twins confirmed LVNC with identical appearances to their mother (Figure 1). Genetic testing identified an alteration in gene MYH7 (c.3908G >C p. (Arg1303 Pro)) in our patient and her monochorionic twins. This genetic change has not been reported before in other individuals.

Conclusion Novel Genes

Our patient is the first reported case study of an alteration in gene MYH7 (c.3908G>C p.(Arg1303 Pro)) in LVNC and this alteration has also been identified in her monochorionic twins.

Pre-pregnancy Counselling

This case demonstrates the importance of pre-pregnancy genetic counselling in inheritable dilated cardiomyopathies.

Multidisciplinary Team Work

Our case illustrates the importance of multidisciplinary team working in complex cardio-obstetric cases to ensure the safest outcome for both mother and neonate. Our team included cardiologists, obstetrics, obstetric anaesthetists, cardiac anaesthetists, cardiac surgeons, referring transplant physicians, midwives, cardiac nurses, neonatologists and clinical genetics.

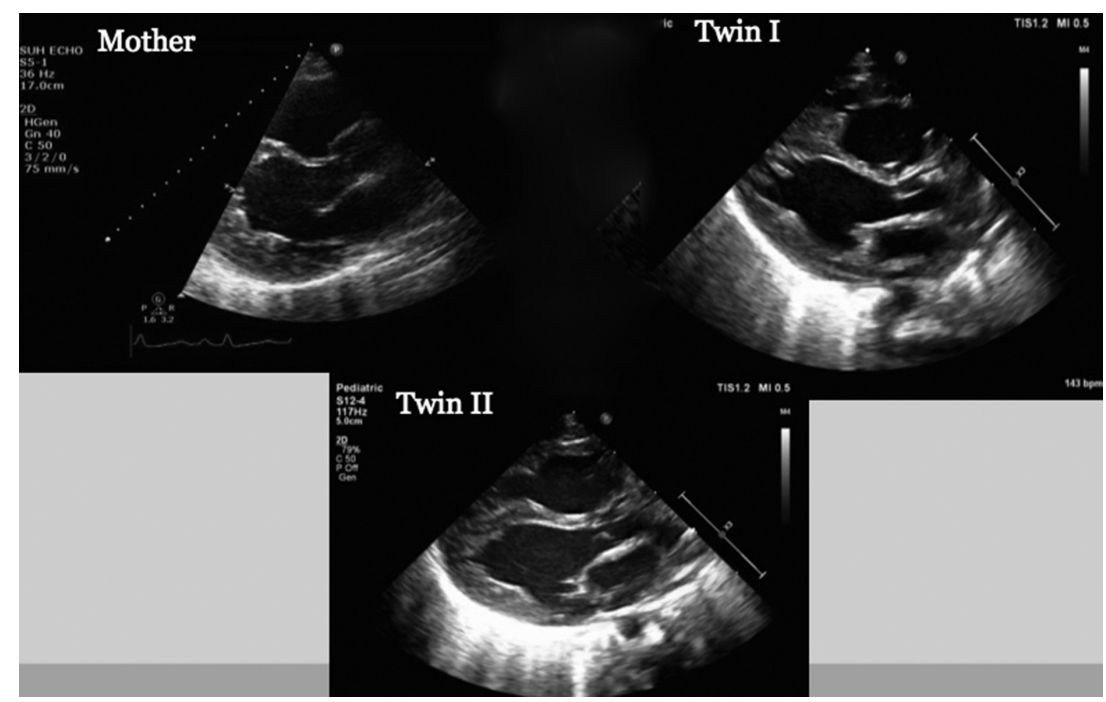

Abstract 75 Figure 1 Plax echocardiographic images of patient (mother) and monochorionic twins 